\title{
Single Layer Cylindrical and Helicoidal Coil With Voids Between Successive Turns Electromagnetic Field Calculation to be Used in Superconductor Current Limiter Simulator for Design Purpose
}

\author{
C. A. Baldan, R. C. Freitas, R. P. Homrich, D. G. Pinatti, E. Ruppert Filho, and C. Y. Shigue
}

\begin{abstract}
A very accurate mathematical method, named Helicoidal Method, to calculate the flux density vector $B$ produced by the current circulating in the resistive single-phase superconductor electrical current limiter (RSCL) coils, at any point of the its whole space, using the Biot-Savart's Law is presented in this paper. It is very important to remember that these coils are single layer concentric cylindrical coils with voids (free space) between its neighbor turns which turns present helicoidal form. The calculation of the vector $B$, at any point of the space of the coils, is used in the dynamic simulation of the RSCL for design and operation purposes, but it is also used to calculate the self-inductance of each electrical current limiter coil and the mutual inductance between each pair of coils, which are necessary to determine the equivalent impedance of the RSCL. To verify the proposed method accuracy the self-inductance of three different coils, made with copper wire, are calculated, measured and the results are compared.
\end{abstract}

Index Terms-Current limiter, electromagnetics, finite elements, superconductor.

\section{INTRODUCTION}

D UE TO ITS electrical and cooling characteristics resistive superconductor electrical current limiters (RSCL) are constituted by single-layer cylindrical coils wound on concentric fiberglass tubes immersed in the cryogenic fluid like liquid helium or nitrogen. The coils are generally parallel connected, present voids between turns and its turns present helicoidal form as it can be seen in [1] and in the Fig. 2 .

When the electrical current circulating in the RSCL coils increases, the magnitude of the magnetic flux density vector B at the superconductor wire surface also increases and a process of transition of the material from the superconductor state to the conductor state (quench) can start. The quench must occur as close as to a previous designed electrical current value. So to

Manuscript received October 21, 2003. This work was funded by FAPESP/Brazil under contract number 1998/6642-2.

C. A. Baldan, D. G. Pinatti, and C. Y. Shigue are with the Chemical Engineering Faculty, Lorena (FAENQUIL), Brazil (e-mail: cabaldan@demar. faenquil.br).

R. C. Freitas and E. Ruppert Filho are with the Systems and Energy Control Department, Computer and Electrical Engineering School, Campinas University (DSCE/FEEC/UNICAMP), Campinas 13081970, Brazil (e-mail: ruppert@fee.unicamp.br).

R. P. Homrich is with the Electrical Engineering Department, Federal University of Rio Grande do Sul (DELET/UFRGS), Rio Grande do Sul State, Brazil (e-mail: homrich@eletro.ufrgs.br).

Digital Object Identifier 10.1109/TASC.2004.830274 design the RSCL it is necessary to use a mathematical dynamic simulator to verify its transient behavior for electrical current variations and design proper coils to assure that the quench will start only at the designed electrical current value as shown in [1].

In the steady-state operation the RSCL must present a very low impedance to the electrical power system which means that the RSCL combined coils must have a very low equivalent impedance when operating in the superconductor state.

So, as the electrical resistance is zero the equivalent inductance must also be very small and this requires an appropriate electrical design including coil self-inductances and mutual inductances between coils accurate calculation method.

As to perform the dynamic simulation of the RSCL transient behavior and also to calculate the inductances of its coils it is absolutely necessary to perform an accurate calculation of the magnetic flux density vector $B$ at each point of the whole space of the RSCL coils including the surface of the wire. This paper presents a very accurate numerical mathematical method to calculate the vector B at any point of the RSCL space using the Biot-Savart's Law considering that the RSCL coils present voids between neighbor turns and that the turns have helicoidal form making the calculation to be very ingenious to give good results. This method will be called Helicoidal Method from now on.

It could be thought to use a finite element software to do this job, but to do that it would be necessary to have the possibility to solve the set of differential equations together with the finite element equations, which would be troublesome and not usual in finite element commercial software package. Another problem with the use of the finite element method could be the presence of voids between the turns of the RSCL coils.

This paper also presents how to calculate RSCL coil self-inductances and mutual inductances between coils. The accuracy of the proposed Helicoidal Method are verified comparing calculated self-inductances values with measured values of three different special coils made with copper wire.

Two types of single-phase RSCL using LTC NbTi superconductor material were designed and constructed using the Helicoidal Method. Both were tested with very good results as shown in [1]. At this time a HTC single-phase RSCL are being designed. Many books and papers [2]-[8] were consulted to find any result that could be used to solve this problem of vector B calculation but in no one it could be found any solution [9]. This 


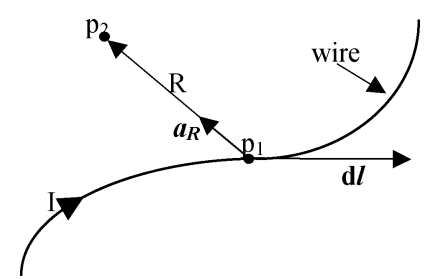

Fig. 1. Biot-Savart's Law illustration for the magnetic flux density vector calculation at the point $\mathrm{p}_{2}$.

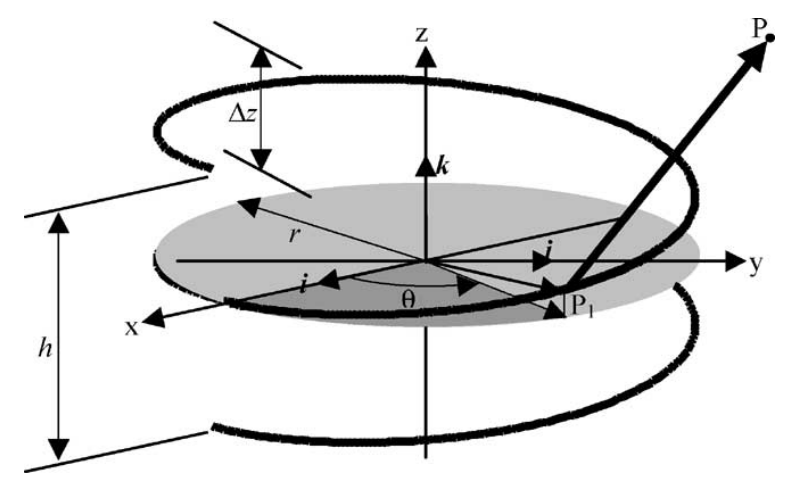

Fig. 2. Helicoidal form winding.

paper presents an improvement of the calculation presented in [9].

\section{THE LAW OF BIOT-SAVART}

At this point it is interesting to review this law in the vector form. Let a wire carrying an electric current $\mathrm{I}$, as illustrated in the Fig. 1.

The calculation of the magnetic flux density vector at the generic point $\mathrm{p}_{2}$ can be done by the application of Biot-Savart's law, represented in the vector form in (1). The direction of the vector $d \boldsymbol{B}_{\mathrm{p}}$ is out of the paper.

$$
d \boldsymbol{B}_{p}=\frac{\mu_{0} \mathrm{Id} \boldsymbol{l} \times \boldsymbol{a}_{\mathrm{R}}}{4 \pi|\mathrm{R}|^{2}} .
$$

\section{The Helicoidal MethoD}

In this calculation method, the coil is helicoidal and the turns describes a helix. In this case it will be taken into account: (a) the existing voids between neighbor turns, (b) the coil height is not necessary larger than coil radius, (c) the magnetic flux density is not uniform neither along the coil axis nor along the coil cross-section area.

Fig. 2 shows a helicoidal winding carrying an electrical current $i$. The magnetic flux density vector $B$ can be determined since the geometrical form of the conductor could be well described mathematically. The point $\mathrm{P}_{1}$ (on the wire) coordinates are defined in the cartesian coordinates according to the wire geometry.

The point $\mathrm{P}$ is located out of the winding and is described generically by the coordinates $\mathrm{x}, \mathrm{y}$ and $\mathrm{z}$ in the cartesian coordinate system. During the subsequent exposition this point will be adequate defined according to the evolution of the winding geometry on which it has the interest to find the vector B.
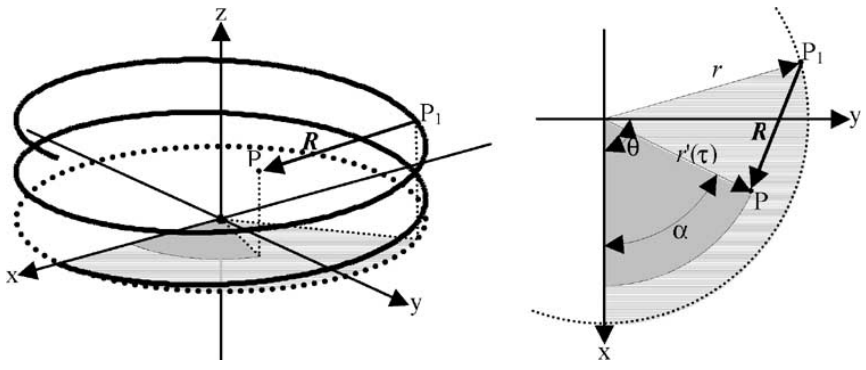

Fig. 3. Angle $\alpha$ definition.

The coil described by the winding mentioned above has $N$ turns, internal radius $r$ and effective height $h$ (concerning the wire position). The turn pitch $\Delta z$ is calculated by (2).

$$
\Delta z=\frac{h}{N} \text {. }
$$

Angle $\theta$ varies from $-\pi N$ to $+\pi N$ with its origin in the $x$ axis of the coordinate system and represents the angular shift of the point $\mathrm{P}_{1}$ that moves along the complete winding. Both $\mathrm{P}$ and $\mathrm{P}_{1}$ are defined, respectively in polar and in the cartesian coordinates as shown in (3) and (4).

$$
\begin{aligned}
& \mathrm{P}_{1}\left(r \cos \theta, r \sin \theta, \frac{\theta h}{2 \pi N}\right), \\
& \mathrm{P}(x, y, z) .
\end{aligned}
$$

For the self-inductance coil calculation the point $\mathrm{P}$, placed in a position determined by the radius $r^{\prime}(\tau)$, will cover all the internal region of the coil $0 \leq r^{\prime}(\tau)<r$.

The point $\mathrm{P}$ coordinates, this way, is defined by (5) and shown in the Fig. 3.

$$
\mathrm{P}\left(r^{\prime}(\tau) \cos \alpha, r^{\prime}(\tau) \sin \alpha, \frac{\alpha h}{2 \pi N}\right) .
$$

Using the Biot-Savart's law for the considered geometry and integrating (1) to get the contribution of all the differential current elements it can have the vector $\mathrm{B}$ components at the generic point $\mathrm{P}$ in cylindrical coordinates as shown in (6), (7) and (8).

$$
\begin{aligned}
B_{r} & =\frac{\mu_{0} \mathrm{i}}{4 \pi} \\
\times & \int_{\theta=-\pi N}^{\theta=+\pi N} \frac{r\left(z-\frac{\theta h}{2 \pi N}\right)(2 \cos \theta-1)+\frac{h}{2 \pi N}(x \sin \theta-y \cos \theta)}{\left[x^{2}+y^{2}+r^{2}-2 r(x \cos \theta+y \sin \theta)+\left(z-\frac{\theta h}{2 \pi N}\right)^{2}\right]^{\frac{3}{2}}} \mathrm{~d} \theta \\
B_{\theta} & =\frac{\mu_{0} \mathrm{i}}{4 \pi} \\
\times & \int_{\theta=-\pi N}^{\theta} \frac{\frac{h}{2 \pi N}[(x \cos \theta-y \sin \theta)-(2 \cos \theta-1) r]}{\left[x^{2}+y^{2}+r^{2}-2 r(x \cos \theta+y \sin \theta)+\left(z-\frac{\theta h}{2 \pi N}\right)^{2}\right]^{\frac{3}{2}}} \mathrm{~d} \theta \\
B_{z} & =\frac{\mu_{0} \mathrm{i} r}{4 \pi} \\
& \times \int_{\theta=+\pi N} \frac{r-(y \sin \theta+x \cos \theta)}{[=-\pi N} \\
&
\end{aligned}
$$




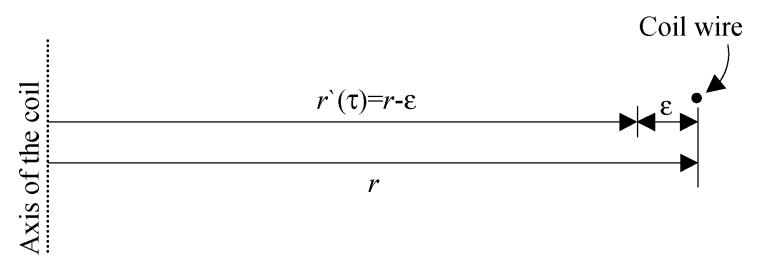

Fig. 4. Representation of $r^{\prime}(\tau) \rightarrow r$ from the left side.

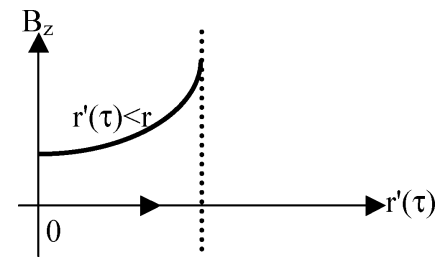

Fig. 5. $\mathrm{B}_{z}$ when $r^{\prime}(\tau) \rightarrow r$ from the left side.

Positioning the point $\mathrm{P}$ as shown in (5) it can write (9), (10), and (11). These equations give the components of the vector $B$, in cylindrical coordinates, at any point $\mathrm{P}$ of the space given in coordinates as shown in (5).

$$
\begin{aligned}
& B_{r}=\frac{\mu_{0} \mathrm{i}}{4 \pi} \\
& \times \int_{\theta=-\pi N}^{\theta=+\pi N} \frac{\frac{r}{2 \pi}\left(\frac{\alpha h}{N}-\frac{\theta h}{N}\right)(2 \cos \theta-1)+\frac{h}{N} r^{\prime}(\tau) \sin (\theta-\alpha)}{\left[r^{\prime}(\tau)^{2}+r^{2}-2 r r^{\prime}(\tau) \cos (\alpha-\theta)+\frac{h^{2}}{4 \pi^{2} N^{2}}(\alpha-\theta)^{2}\right]^{\frac{3}{2}}} \mathrm{~d} \theta,
\end{aligned}
$$

$B_{\theta}=\frac{\mu_{0} \mathrm{i}}{4 \pi}$

$$
\times \int_{\theta=-\pi N}^{\theta=+\pi N} \frac{\frac{h}{2 \pi N}\left[r^{\prime}(\tau) \cos (\alpha-\theta)-(2 \cos \theta-1) r\right]}{\left[r^{\prime}(\tau)^{2}+r^{2}-2 r r^{\prime}(\tau) \cos (\alpha-\theta)+\frac{h^{2}}{4 \pi^{2} N^{2}}(\alpha-\theta)^{2}\right]^{\frac{3}{2}}} \mathrm{~d} \theta,
$$

$$
\begin{aligned}
B_{z} & =\frac{\mu_{0} \mathrm{i} r}{4 \pi} \\
& \times \int_{\theta=+\pi N} \frac{r-r^{\prime}(\tau) \cos (\alpha-\theta)}{\left[r^{\prime}(\tau)^{2}+r^{2}-2 r r^{\prime}(\tau) \cos (\alpha-\theta)+\frac{h^{2}}{4 \pi^{2} N^{2}}(\alpha-\theta)^{2}\right]^{\frac{3}{2}}} \mathrm{~d} \theta .
\end{aligned}
$$

The $\mathrm{z}$ component of the vector $B\left(B_{\mathrm{z}}\right)$, that is used for coil linkage magnetic flux calculation is a noncontinuous function for $r^{\prime}(\tau)=r$. During the mathematical numerical integration for the purpose of the coil linkage flux calculation, the angle $\theta$ is continuously incremented while the angle $\alpha$ is kept constant. Consequently, depending on the discretization used for the increment of $\theta$ it can occur situations where $\theta=\alpha$. In this case the denominator of (9), (10) and (11) is reduced to $r^{\prime}(\tau)^{2}+r^{2}-2 r r^{\prime}(\tau)$.

If $r^{\prime}(\tau)$ tends to $r, B_{z}$, in (12), tends to extremely high values.

$$
B_{z}=\frac{\mu_{0} i r N}{2} \frac{r-r^{\prime}(\tau)}{\left[r^{\prime}(\tau)^{2}+r^{2}-2 r r^{\prime}(\tau)\right]^{\frac{3}{2}}} .
$$

If $r^{\prime}(\tau)$ goes to the limit of $r$, writing $r^{\prime}(\tau)=r-\varepsilon$, doing $\varepsilon$ to tend to zero, it has the situation shown in the Figs. 4 and 5.

$$
\begin{aligned}
& \lim _{\varepsilon \rightarrow 0} B_{z}=\frac{\mu_{0} \mathrm{i} r N}{2} \lim _{\varepsilon \rightarrow 0} \frac{r-(r-\varepsilon)}{\left[(r-\varepsilon)^{2}+r^{2}-2 r(r-\varepsilon)\right]^{\frac{3}{2}}}, \\
& \lim _{\varepsilon \rightarrow 0} B_{z}=\infty .
\end{aligned}
$$

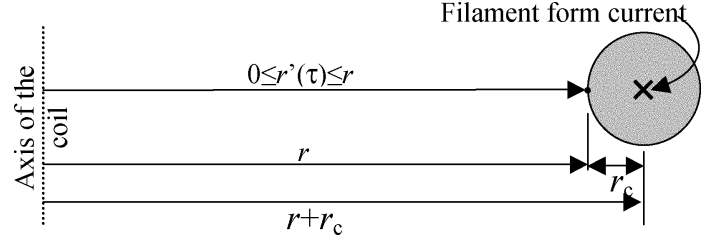

Fig. 6. Representation of the value $r^{\prime}(\tau) \rightarrow r$.

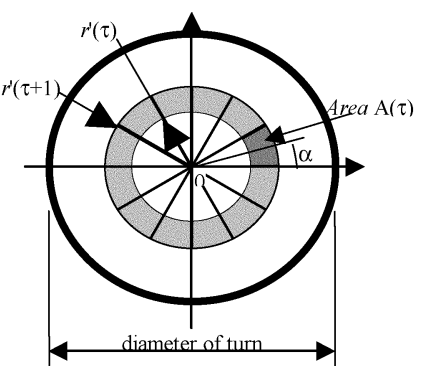

(a)

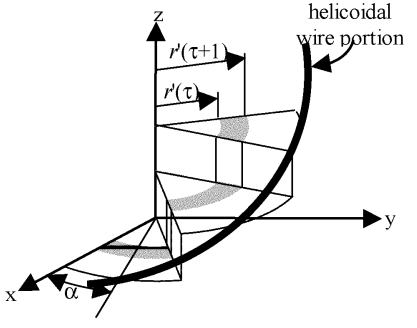

(b)
Fig. 7. Discretization of the radius of the coil in rings (a) and sectors (b).

One way to avoid the excessive $r^{\prime}(\tau)$ approximation to the value $r$ is to consider that the electrical current has a filament form and flows in the center of the conductor. So in between the filament form current and the surface of the conductor there will be a distance corresponding to the radius of the conductor, $r_{\mathrm{c}}$, as shown in the Fig. 6.

\section{Calculation of the Coil SElf-Inductance}

To perform this calculation it is necessary to use the magnitude of $B_{\mathrm{z}}$ at any point of the coil space, as calculated before, to calculate the flux linkage with each one of the turns. In the case of plane turns this calculation is not difficult because $B_{\mathrm{z}}$ is perpendicular to the turn area but in the case of helicoidal turns this situation does not occur. Although $B_{\mathrm{z}}$ be in the same direction of the turn axis, the direction of the area vector changes with the inclination of the turn. In this case what is done to calculate the flux linking each helicoidal turn is to divide each turn in rings and sectors as shown in the Figs. (7a) and (7b), respectively.

Each sector area is done by (15), where $s$ is the number of sectors of each ring.

$$
\operatorname{Area}(\tau)=\frac{\pi}{s}\left\{\left[r^{\prime}(\tau+1)\right]^{2}-\left[r^{\prime}(\tau)\right]^{2}\right\} .
$$

The magnetic flux crossing each sector is shown in (16).

$$
\phi_{z}(\alpha, \tau)=\operatorname{Area}(\tau) \frac{B_{z}(\alpha, \tau)+B_{z}(\alpha, \tau+1)}{2} .
$$

In (16) $\alpha$ is the angle of the average point of the sector (rad).

The magnetic flux crossing the turn $k$ of the coil is given by (17).

$$
\phi_{k}=\sum_{\tau=1}^{d} \sum_{v=1}^{2 s} \phi(\alpha, \tau)
$$

$\alpha=v(\pi / s)$; with $v$ odd and $\alpha$ in $\operatorname{rad}$.

The coil linkage flux is given by (18).

$$
\lambda=\sum_{k=1}^{N} \phi_{k}
$$


TABLE I

COILS $\mathrm{C}_{1}, \mathrm{C}_{2}$ AND $\mathrm{C}_{3}$ DATA

\begin{tabular}{lccc}
\hline \hline & \multicolumn{3}{c}{ Coil Identification } \\
& $\mathrm{C}_{1}$ & $\mathrm{C}_{2}$ & $\mathrm{C}_{3}$ \\
\hline number of turn & 5 & 10 & 20 \\
coil length $(h), \mathrm{mm}$ & 37.5 & 75 & 150 \\
Internal coil radius $(r), \mathrm{mm}$ & 150 & 150 & 150 \\
wire radius, mm & 0.25 & 0.25 & 0.25 \\
wire length, $\mathrm{m}$ & 4.71 & 9.42 & 18.84 \\
voids between turns, $\mathrm{mm}$ & 7 & 7 & 7 \\
calculated inductance, $\mathrm{mH}$ & 0.0168 & 0.0488 & 0.1354 \\
measured inductance, $\mathrm{mH}$ & 0.0165 & 0.0480 & 0.1337 \\
error, \% & 1.81 & 1.66 & 1.27 \\
\hline \hline
\end{tabular}

Substituting (17) in (18), it has the coil linkage flux as can be seen in (19).

$$
\lambda=\sum_{k=1}^{N} \sum_{\tau=1}^{d} \sum_{v=1}^{2 s} \phi_{z}(\alpha, \tau) .
$$

The coil self-inductance is calculated by (20).

$$
\mathrm{L}=\lambda i^{-1} \text {. }
$$

In the same way as described before it is possible also to calculate all the mutual inductances between turns. It is necessary to say at this point that the inductances could be also calculated using the Neumann's formula as well as Weber's formula, Maxwell's formula or Graneau's formula [10], that are energy formulas, but as the $B$ vector must be calculated at each point of the coils, for limiter design purpose, this way the presented method was preferred because it saves computational time.

\section{EXPERIMENTAL RESULTS}

To validate the presented mathematical method, a computer program was implemented in Turbo Pascal language for the numeric calculation of the integrals. To check the calculated values, the self-inductances of three special made coils $\mathrm{C}_{1}, \mathrm{C}_{2}$ and $\mathrm{C}_{3}$ were measured. The data of each coil, the calculated and measured self-inductances of each one of them are presented in the Table I. To measure the self-inductances it was used a using a GenRad 1659 RLC Didibridge. As it can be seen in the Table I the errors calculated using (21) are less than $2 \%$ and can be considered very small.

On the other hand this method is computationally very time consumption requiring about 1 hour and 20 minutes to calculate the self-inductance of the coil $\mathrm{C}_{2}$ and about 5 hours to calculate the self-inductance of the coil $\mathrm{C}_{3}$.

error $=\left[\frac{\text { calculated inductance }- \text { measured inductance }}{\text { measured inductance }}\right] \cdot 100 \%$

This proposed method was used to design two different types (type $\mathrm{W}$ and type O) LTC RSCL reported in [1] with absolute success.

\section{CONCLUSIONS}

A very accurate numerical mathematical method was developed to calculate the magnetic flux density vector at any point of a RSCL coils and also all the coils self-inductances and mutual inductances between coils taking into account the complete geometry of the coils. The accuracy of the method was verified comparing the measured self-inductance of three coils especially made with copper wire with the calculated self-inductances using the method. The result was really very accurate but the computational time consumed was very high suggesting that other methods must be searched so as to decrease the computational time.

However the method is recommended to be used in the design phase of the RSCL if it can have adequate computational resource.

\section{REFERENCES}

[1] R. P. Homrich, E. Ruppert Filho, D. G. Pinatti, C. A. Baldan, and C. Y. Shigue, "Single-phase resistive superconductor electrical current limiter," IEEE Trans. Appl. Supercond., vol. 12, no. 1, pp. 1386-1389, March 2001.

[2] J. D. Kraus, Electromagnetics. New York: McGraw-Hill Inc., 1984.

[3] S. B. Hammond, Electrical Engineering. New York: McGraw-Hill Inc., 1961.

[4] W. H. Hayt Jr., Engineering Electromagnetics. New York: McGrawHill, Inc., 1981.

[5] J. R. Reitz, F. J. Milford, and R. W. Christy, Foundations of Electromagnetic Theory. New York: Addison Wesley Publishing, Inc., 1980.

[6] S. Ramo, J. R. Whinnery, and T. Van Duth, Fields and Waves in Communication Electronics. New York: Wiley Int., 1965.

[7] D. D. Cheng, Field and Waves Electromagnetics. New York: Addison Wesley Publishing, Inc., 1989.

[8] T. L. Simpson, "Effect of a conducting shield on the inductance of an air-core solenoid," IEEE Trans. Magnetics, vol. 35, no. 1, pp. 508-515, January 1999

[9] R. P. Homrich, E. Ruppert Filho, and D. G. Pinatti, "Helicoidal singlelayer cylindrical oil self-inductance evaluation: a didatic method," IEEE Trans. Education, vol. 44, p. 202 (enclosed CD-ROM), May 2001.

[10] M. Bueno and A. K. T. Martins, "Equivalence between the formulas for inductance calculation," Can. J. Phys., vol. 75, pp. 357-362, 1997. 